Article

\title{
In vivo Modelling of Toxicity of Eight Commercial Artificial Sweeteners in Daphnia Neonates and Zebrafish Embryos through Cardiac Performance
} Assessments

\author{
Ferry Saputra ${ }^{1 \sharp}$, Yu-Heng Lai ${ }^{2}$, Rey Arturo T. Fernandez ${ }^{3}$, Allan Patrick G. Macabeo ${ }^{3}$, Hong- \\ Thih Lai ${ }^{*}$, Jong-Chin Huang ${ }^{*}$ and Chung-Der Hsiao ${ }^{1,6,7 *}$ \\ 1. Department of Bioscience Technology, Chung Yuan Christian University, Chung-Li, 32023, Taiwan \\ 2. Department of Chemistry, Chine se Culture University, Taipei11114, Taiwan \\ 3. Laboratory for Organic Reactivity, Discovery and Synthesis (LORDS), Re search Center for the Natural \\ and Applied Sciences, University of Santo Tomas, Espana St., Manila 1015, Philippines \\ 4. Department of Aquatic Biosciences, NationalChiayi University, 300 Syue fu Rd. Chiayi, 600 Taiwan \\ 5. Department of Applied Che mistry, National Pingtung University, Pingtung, 90003, Taiwan \\ 6. Department of Bioscience Technology, Chung Yuan Christian University, Chung-Li32023, Taiwan \\ 7. Center for Nanotechnology, Chung Yuan Christian University, Chung-Li32023, Taiwan \\ \# equal contribution authors \\ * Correspondence: Correspondence: Hong-Thih Lai(htlai@mail.ncyu.edu.tw); Jong-Chin Huang \\ (hjc@mail.nptu.edu.tw); and Chung-Der Hsiao (cdhsiao@cycu.edu.tw)
}

\begin{abstract}
Artificial sweeteners are widely used food ingredients in beverages and drinks to lower calorie intake which in turn prevent lifestyle diseases such as obesity. Epidemiological evidences suggest that an overdose of artificial sweeteners could result to adverse effects after consumption. Thus, our study aims to systematically explore the potential adverse effects of eight commercial artificial sweeteners, including acesulfame-K, alitame, aspartame, sodium cyclamate, dulcin, neotame, saccharin and sucralose on cardiac performances of zebrafish (Danio rerio) and Daphnia as model animals. Embryonic zebrafish and Daphnia were exposed to eight artificial sw eeteners at 100 $\mathrm{ppb}$ concentrations and their cardiac performance (heart rate, ejection fraction, fractional shortening, stroke volume, cardiac output and heartbeat regularity) were measured and compared. Saccharin significantly increased the heart rate of zebrafish larvae while a significant decrease was observed in Daphnia. Significant increase was also noted in zebrafish heart rate variability after incubation in acesulfame K, dulcin, sodium cyclamate, and sucralose. However, a significant increase in Daphnia was only observed after incubation in dulcin. Based on Principal Component Analysis (PCA) and hierarchical clustering results, several artificial sweetener samples were species-specific to zebrafish and Daphnia. Our study demonstrates the potential adverse physiological effects of artificial sweeteners in cardiovascular systems of zebrafish larvae and Daphnia.
\end{abstract}

Keywords: Artificial sweeteners; zebrafish; Daphnia; cardiac performance; toxicity

\section{Introduction}

The intake of sugar-sweetened beverages is popularly associated with lifestyle-related diseases such obesity, type 2 diabetes, and a number of metabolic syndromes [1]. Based on the above disease associations, scientists and healthcare practitioners theorized that non-caloric, high-intensity sweeteners may provide a beneficial alternative in food and beverages. Thus, non-caloric sw eeteners replacing natural caloric sweeteners reduces energy density of foods and beverages. However, 
whether decreasing energy density in this practice would propel into reduced energy intake, lower body weight, and improved metabolic health is subject to question.

Artificial sweeteners, the non-caloric alternative to sugar, has ironically been linked to consumption of more calories for its ability to increase sugar cravings and dependence as well as impairing caloric compensation leading to appetite stimulation [2]. Independent from this mechanism, artificial sw eeteners are associated with impaired glucose tolerance secondary to altered gut microbiota $[3,4]$. These are some of the proposed mechanisms that despite artificial sw eeteners being non-caloric are associated with obesity, type 2 diabetes mellitus and cardiovascular diseases [5]. These associated life-style related diseases are clearly linked to the development of the atherosclerotic plaque which may cause ischemia and acute coronary syndrome [6,7]. How ever, we theorize that artificial sw eeteners may also induce direct cardiac toxicity and damage independent of plaque formation. This is supported by studies done on Wistar albino rats wherein aspartame induces oxidative stress in cardiac muscle and increases heart rate variability [8,9]. Study done in aquatic animal in also showed that aspartame also cause oxidative stress in brain, gills and muscles of common carp [10]. Related diseases and effects of artificial sw eetener exposure in human and some animal models are listed below (Table 1)

Table 1. Study of related dise ase and effect on biomarker after artificial sweetener exposure.

\begin{tabular}{|c|c|c|}
\hline & Related disease & Effect on biomarker \\
\hline Human & $\begin{array}{l}\text { Obesity, diabetes, hypertension, } \\
\text { cardiovascular event [11], } \\
\text { hepatotoxicity [12], nausea, vomiting, } \\
\text { thrombocytopenia [13]. }\end{array}$ & $\begin{array}{l}\text { Lactate dehydrogenase } \uparrow[14] \\
\text { Acetylcholinesterase } \downarrow[15] .\end{array}$ \\
\hline Rodent & $\begin{array}{l}\text { Obesity, gut biota community shift } \\
\text { [4], vascular endothelial dysfunction } \\
\text { [16], glucose intolerance [17], thy roid } \\
\text { tumor [18] (Rat). }\end{array}$ & $\begin{array}{l}\text { Dopamine } \uparrow \text {, hydroxytryptamine } \uparrow \text {, } \\
\text { norepinephrine } \uparrow \text {, epinephrine } \uparrow \text { [19], } \\
\text { Xanthine oxidase } \uparrow \text {, superoxide dismutase } \uparrow \\
\text {, catalase } \uparrow[8] \text { (Rat). }\end{array}$ \\
\hline Fish & $\begin{array}{l}\text { Swimming defect, inflammatory in } \\
\text { brain and liver [20], and grow th } \\
\text { malformation [21] (Zebrafish). }\end{array}$ & $\begin{array}{l}\text { Reactive oxygen species } \uparrow \quad[20] \text { (Zebrafish), } \\
\text { superoxide dismutase } \uparrow \text {, catalase } \uparrow, \text { lipid } \\
\text { peroxidase } \uparrow[10] \text { (Common Carp). }\end{array}$ \\
\hline Daphnia & No (directly linked to mortality). & $\begin{array}{l}\text { Lipid peroxidation } \uparrow, \operatorname{AChE} \uparrow \text {, antioxidant } \\
\text { capacity } \uparrow[22] \text {. }\end{array}$ \\
\hline
\end{tabular}

$* \uparrow$ mean increase and $\downarrow$ mean decrease.

Understanding the pathophysiologic mechanism of artificial sweeteners on cardiac toxicity requires models wherein the parameters of interest are easily measured but at the same time represent the complexity of the human heart. Danio rerio, also known as the zebrafish, is a commonly used vertebrate model in physiologic, genetic and regenerative experiments on cardiac diseases [2326]. Among the vertebrate species, it is particularly favored because of its relatively short life span which allows investigators to monitor the disease in an accelerated phase [27]. Another organism, Daphnia magna, is an established invertebrate model for the investigation of toxins in water in ecologic systems $[28,29]$. This invertebrate can also be used as a model for cardiovascular diseases for it has a myogenic heart which exhibits responses comparable to the human heart [30].

As part of our interests to provide in vivo models to study the effects of substances in a variety of disease model systems [31-33], zebrafish and daphnia were explored as animal host models to investigate cardiac performance after exposure to eight different artificial sweeteners by measuring different cardiac parameters such as the stroke volume, cardiac output, ejection fraction, and shortening fraction, along with heart rate, heartbeat interval, and heart rate variability.

\section{Materials and Methods}




\subsection{Animal ethics and artificial sweetener exposure}

All protocols and procedures involving zebrafish were approved by the Committee for Animal Experimentation of the Chung Yuan Christian University (Approval No. 109001, issue date 15 January 2020). In this study, wild type AB strain zebrafish was used as a vertebrate model and maintained in a continuously filtered and aerated water system. Temperature was maintained at 26 ${ }^{\circ} \mathrm{C}$ with a 10/14 hours of dark/light cycle according to previously reported protocols [34]. The eggs were collected and kept in an incubator at $28^{\circ} \mathrm{C}$ until the time of treatment. At 60 hour-postfertilization (hpf), approximately fifteen zebrafish larvae were exposed to $20 \mathrm{~mL}$ volume of artificial sweeteners at $100 \mathrm{ppb}$ doses in a $9 \mathrm{~cm}$ Petri dish. At $72 \mathrm{hpf}$, zebrafish heartbeat at the vent ricle chamber was recorded (Fig. 1A). D. magna was used as an invertebrate model. Female Daphnia neonates were collected when body size grew around $0.7-2 \mathrm{~mm}^{2}$ and exposed to artificial sw eeteners at $100 \mathrm{ppb}$ concentration at $26^{\circ} \mathrm{C}$ for 24 hours. After incubation, the heartbeat at the heart chamber of Daphnia was also recorded (Fig. 1B).

In this study, eight artificial sweeteners acesulfame $\mathrm{K}$, alitame, aspartame, dulcin, neotame, saccharin, sodium cyclamate, and sucralose were tested for their potential cardiotoxicity in zebrafish. All of the artificial sw eeteners were purchased from Aladdin chemicals (Aladdin, Shanghai, China). Stock solution of artificial sweeteners was prepared with distilled water and diluted to a stock concentration of $1000 \mathrm{ppm}$. During the assay, stock solutions were diluted to a testing concentration of $100 \mathrm{ppb}$ and applied to embryonic zebrafish and Daphnia (Table 2The assay concentration $100 \mathrm{ppb}$ was show $n$ to be the low est concentration that can alter cardiac parameters based on our pre-testing protocols. MS222 (Tricaine methanesulfonate) and glucose were used as negative and positive control, respectively. MS222 has been used as anesthetic for zebrafish and Daphnia, and is known to decrease cardiac performance $[35,36]$ while glucose know $n$ to increase cardiac parameter $[37,38]$. The experiment $\mathrm{w}$ as done in triplication with average of five individuals per compound.

Table 2. Mole cular formula and acute toxicity information of the eight artificial sweeteners.

\begin{tabular}{cccc}
\hline Number & Artificial sweetener & Molecular formula & Aquatic acute toxicity \\
\hline 1 & acesulfame $\mathrm{K}$ & $\mathrm{C}_{4} \mathrm{H}_{4} \mathrm{KNO}_{4} \mathrm{~S}$ & LC50: $96 \mathrm{Hr}$ for Fish: (mg/L): > \\
& & & 1000 \\
\hline 2 & alitame & $\mathrm{C}_{14} \mathrm{H}_{25} \mathrm{~N}_{3} \mathrm{O}_{4} \mathrm{~S}$ & N.A. \\
\hline 3 & aspartame & $\mathrm{C}_{14} \mathrm{H}_{18} \mathrm{~N}_{2} \mathrm{O}_{5}$ & N.A. \\
\hline 4 & dulcin & $\mathrm{C}_{9} \mathrm{H}_{12} \mathrm{~N}_{2} \mathrm{O}_{2}$ & N.A. \\
\hline 5 & neotame & $\mathrm{C}_{20} \mathrm{H}_{30} \mathrm{~N}_{2} \mathrm{O}_{5}$ & N.A. \\
\hline 6 & saccharin & $\mathrm{C}_{7} \mathrm{H}_{5} \mathrm{NO}_{3} \mathrm{~S}$ & N.A. \\
\hline 7 & sodium cyclamate & $\mathrm{C}_{6} \mathrm{H}_{12} \mathrm{NO}_{3} \mathrm{SNa}_{2}$ & N.A. \\
\hline 8 & sucralose & $\mathrm{C}_{12} \mathrm{H}_{19} \mathrm{Cl}_{3} \mathrm{O}_{8}$ & N.A. \\
\hline
\end{tabular}

WHO GHS acute aquatic toxicity definition is $96 \mathrm{hr}$ LC50 less than $1 \mathrm{ppm}$ for fish or $48 \mathrm{hr}$ EC50 less than 1ppm for Crustaceans. N.A. not available.

\subsection{Cardiac Performance measurement}

To record zebrafish heart beats and cardiac physiology parameters, $3 \%$ methyl cellulose was used as a mounting agent to minimize zebrafish movement during video recording. High-speed digital charged coupling device (CCD) (AZ Instrument, Taichung, Taiw an) w as mounted on inverted microscope (Sunny Optical Technology, Zhejiang, China) to record zebrafish heartbeat. To acquire better image contrasts and resolutions, Hoffmann objective lens with 40x magnification were used, and video was recorded at 200 frames per second (fps) for 10 second [39]. Similar settings were also used to record Daphnia heart with LPlan Lens with 20x magnification to record heart rate. To calculate cardiac parameters, Time Series Analyzer V3 plug-in (https://imagej.nih.gov/ij/plugins/timeseries.html) on ImageJ softw are w as used to analyze the pattern of changes in dynamic pixel intensity $[40,41]$. Heart rate, expressed as beats per minute (b pm), was measured using the Peak analyzer function in OriginPro 2019 software (Originlab Corporation, Northampton, MA, USA) by 
determining the time interval of each peak. The Poincare Plot was generated using a Poincare plugin from the OriginPro 2019 software. Sd1 and sd2 extracted from the plots were recorded and statistically analyzed to calculate heart rate. Stroke volume is determined by the assumption that heart chamber have ellipsoid shape and calculated by subtracting end-systolic volume (ESV) from the end-diastolic volume (EDV). The volume of the heart chamber was calculated using the heart chamber long (DL) and short axis (Ds) to compare the volume difference between EDV and ESV [42]. Cardiac output was calculated by multiplying heart rate observed in the ventricle with stroke volume. Ejection fraction and shortening fraction was calculated using the formula:

$$
\begin{gathered}
E F(\%)=\frac{S V}{E D V} \times 100 \% \\
S F(\%)=\frac{D s(E D V)-D s(E S V)}{D s(E D V)} \times 100 \%
\end{gathered}
$$

A.

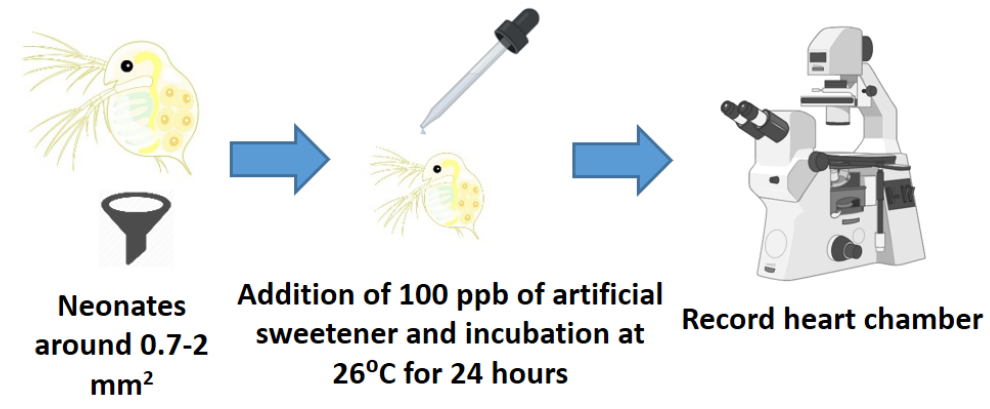

B.

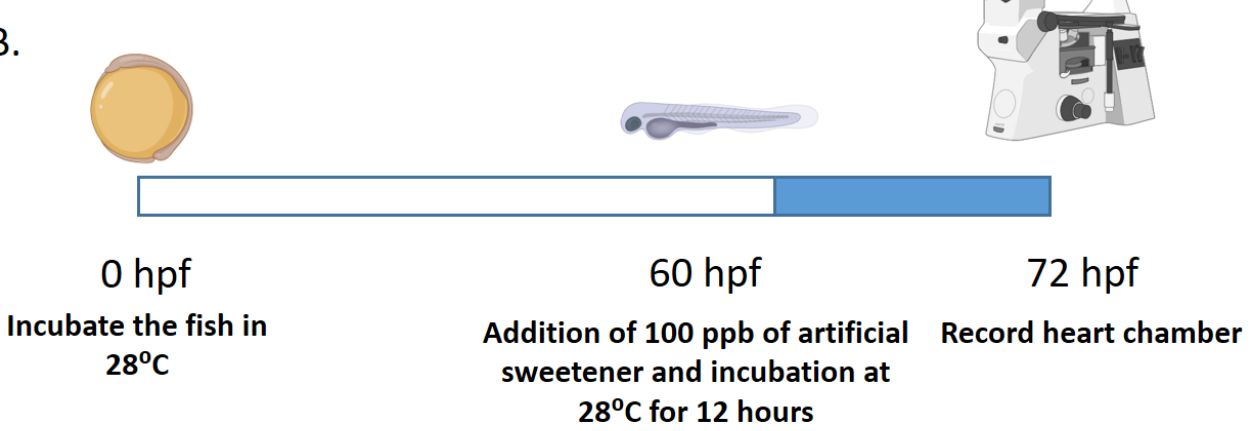

Figure 1. Schematic dia gram showing experimental de sign on te sting cardiotoxicity of eight artificial sweeteners in Daphnia ne ona tes (A) and ze bra fish e mbryos (B).

\subsection{Principal component analysis (PCA) and hierarchy clustering}

Principal component analysis (PCA) and the heat map are two methods that able to reduce data complexity and perform data grouping. PCA and heat map were constructed using a ClustVis on line tool (a w eb tool for visualizing clustering of multivariate data, https://biit.cs.ut.ee/clustvis/) with default setting [43]. To obtain accurate clustering, data were normalized with the control and mean percentage was calculated.

\subsection{Biostatistics}

Statistical analysis w as done by latest GraphPad Prism (GraphPad Inc., La Jolla, CA, USA). To determine the significance, non-parametric One-w ay ANOVA (Kruskal-Wallis test) was performed and the level of significance $w$ as set at $p$ value $<0.05$.

\section{Results}

3.1. Cardiac performance in zebrafish and Daphnia after exposed to artificial sweeteners 
To assess cardiac performance, heart rate, cardiac interval, stroke volume, cardiac output, ejection fraction, and shortening fraction w ere analyzed using ImageJ soft ware. Heart rate variability was calculated using a Poincare Plot to check regularities of each beat after exposure to different artificial sw eeteners [44].

The cardiac cycle is divided into two phases: systole and diastole [45]. Increases in heart rate affect diastole by decreasing the time that it takes for blood to fill the ventricles. Conversely, at slower heart rates, left ventricular end diastolic volume is larger. It is also during diastole that blood flows to the coronary arteries to supply the heart [46]. Increases in heart rate will decrease coronary perfusion time and this is problematic in patients with coronary stenosis for it can lead to tissue ischemia. Furthermore, elevation of heart rate adversely affects myocardial oxygen balance by increasing the demand for oxygen [47]. Increases in heart rate also adversely affects patients with pulmonary hypertension since it w orsens biventricular function [48]. Hence, artificial sw eeteners that cause increase in heart rate should not be given to patients with coronary artery stenosis for it will further lead to tissue ischemia.

Heart rate can be affected by-hormones, temperature, or by exogenous compounds [49]. At 100 ppb test concentration, acesulfame $\mathrm{K}$, neotame, saccharin, and sucralose significantly increased zebrafish heart rate compared to the control group (Fig. 2A). On the other hand, saccharin significantly decreased heart rate in Daphnia (Fig. 2B).

Low time intervals, as noted in increased heart rate, result in a reduced stroke volume secondary to a decrease in ventricular filling. Humans, particularly athletes used to high intensity exercise, will manifest a longer time interval between each beat allowing ventricles to fill with blood efficiently [50]. After incubation with $100 \mathrm{ppb}$ test concentration of acesulfame $\mathrm{K}$, neotame, saccharin, and sucralose in zebrafish larvae, a significant decrease in each beat was observed (Fig. 2A). In Daphnia, significant increase in time interval was noted after incubation in $100 \mathrm{ppb}$ of sucralose(Fig. 2B).

To measure the amount of blood pumped during systole, stroke volume was also determined [39]. Stroke volume was calculated by subtracting the volume of the heart chamber at end diastolic phase (EDV) with the heart chamber volume at end systolic phase (ESV). No significant change in zebrafish stroke volume after incubation in all compounds was observed (Fig. 2A). However, a significant decrease was observed after incubation in alitame and dulcin in Daphnia (Fig. 2B).

Cardiac output, an important end point in cardiovascular system assessment, was determined by multiplying heart rate and stroke volume [51]. Thus, incubation in acesulfame K, alitame, aspartame, dulcin, and sucralose slightly elevated cardiac output in zebrafish compared to the control group (Fig 2A). The opposite was true in Daphnia, where significant decrease in cardiac output compared to the control group after incubation in alitame, dulcin, and saccharine was observed (Fig. 2B).

Ejection fraction was likewise calculated by dividing stroke volume from EDV [52]. No significant change in the ejection fraction among the different artificial sweeteners used on either zebrafish or Daphnia compared to the control were observed (Fig. 2A and 2B).

Finally, the index of shortening fraction was determined by obtaining the rat io of the length of heart chamber at the end of systolic phase to end of diastolic phase, which represents muscular contractility of the heart [52]. The artificial sweetener sodium cyclamate significantly decreased the shortening fraction in zebrafish (Fig. 2A). In contrast, all other sweeteners decreased the shortening fraction in Daphnia (Fig. 2B). 


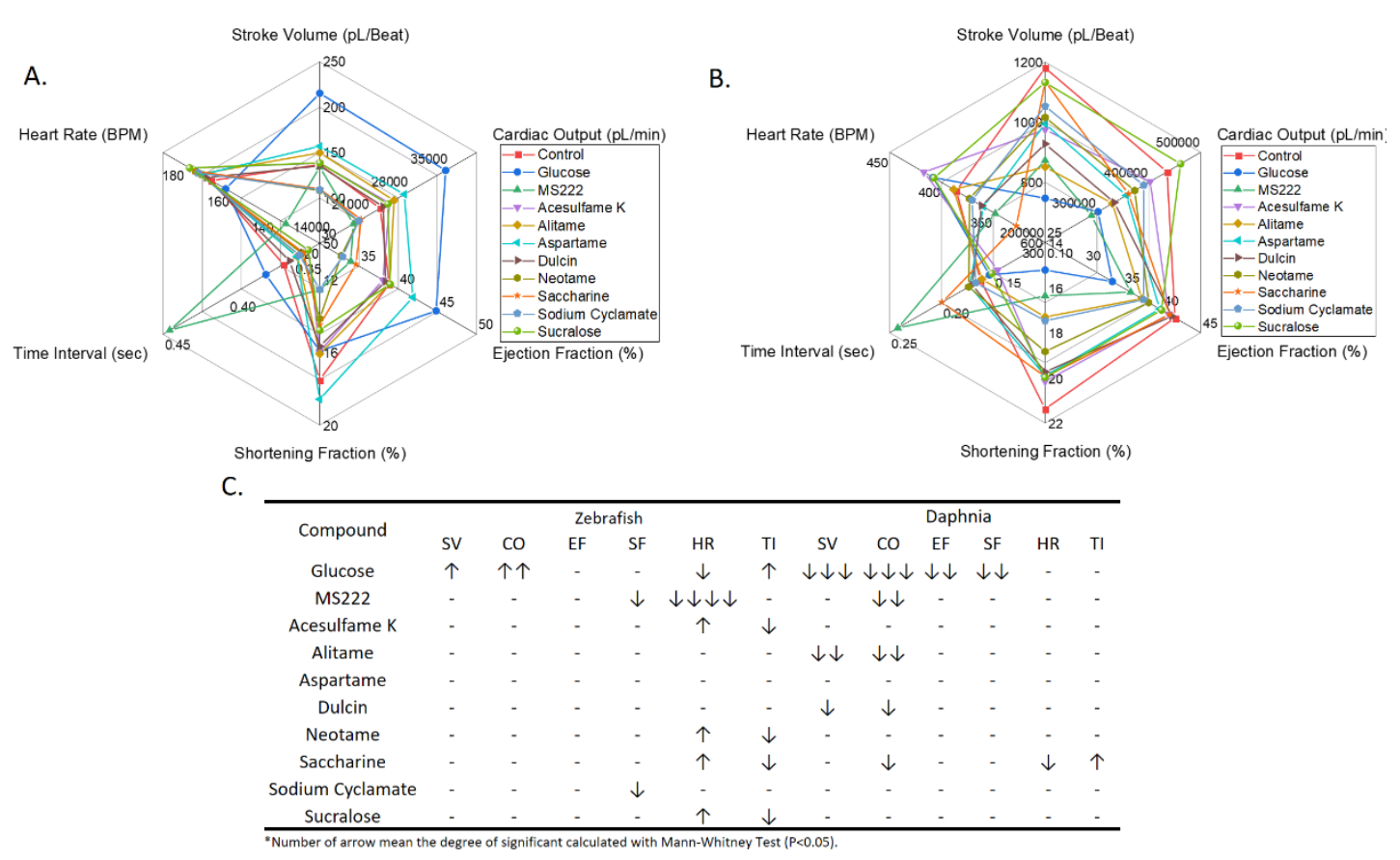

Figure 2. Ra diac ma p comparing the mean value of cardiac performance endpoints in either zebrafish (A) or Daphnia (B) after exposure to eight diffe rent artificial sweeteners. (C) The degree of significance of each cardiac performance endpoints in either zebrafish or Daphnia after exposure to eight artificial sweeteners. SV, stroke volume; CO, cardiac output; EF, ejection fractioning; SF, shortening fraction; $\mathrm{HR}$, he art rate and TI, time interval.

\subsection{Heart rate regularity in zebrafish and Daphnia after exposure to artificial sweeteners}

Using Poincare plot, the artificial sw eeteners w ere checked whether they confer alteration in the variability of heart rate. Poincare plot is a method that plots the heartbeat interval between two successive heart beats and has been used to study heart rate regularity in humans, rodents and zebrafishes [53-56]. A higher standard deviation means that the heartbeat is more irregular. Thus, incubation of zebrafish larvae in acesulfame K, dulcin, saccharin, and sodium cyclamate significantly increased sd1 but show ed no significant difference with sd2 (Fig. 3A\&B). While slightly elevation of heart rate variability after incubation in neotame, sodium cyclamate, and sucralose in Daphnia was observed, however not reach statistical significance. Among eight artificial sw eeteners tested, only dulcin significantly increased the heart rate variability with significant higher sd1 value in Daphnia (Fig. 3C \& D). 


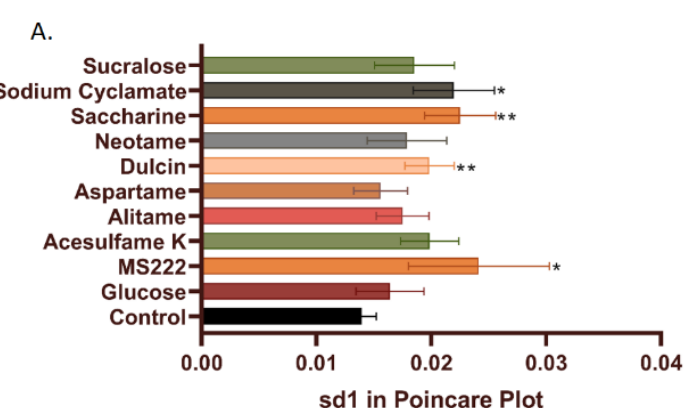

B.

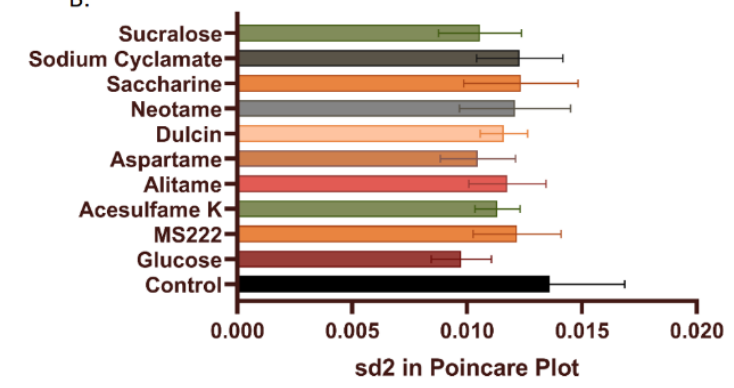

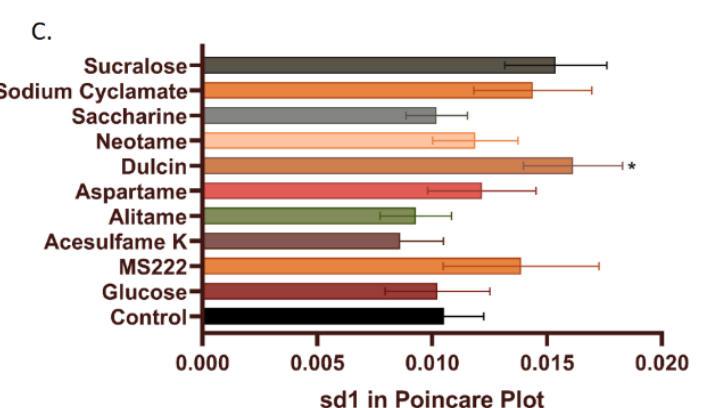

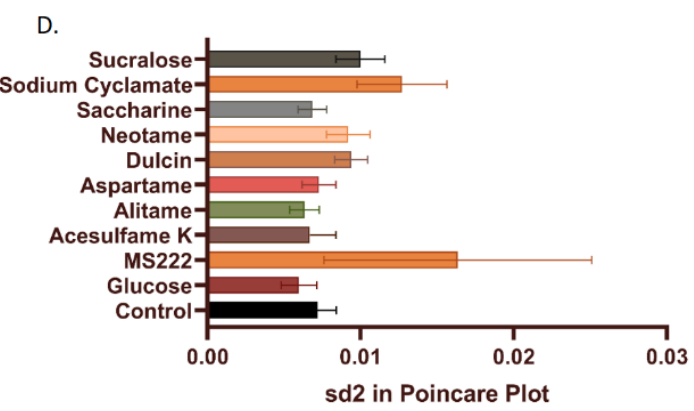

Figure 3. Comparison of heart rate regularity in zebrafish embryos (A\&B) and Daphnia (C\&D) after incubation eight different artificial sweeteners. Data analysis was based on the standard deviation generated by Poinca re Plot, and presented as mean \pm SEM and statistical significances we re tested by non-para metric One-Way ANOVA (Kruskal-Wallis test) with post-hoc analyzed. ${ }^{*}, \mathrm{p}<0.05$; ${ }^{* *}, \mathrm{p}<0.01$.

\subsection{Comparison of cardiac performance between zebrafish and Daphnia after exposure to artificial sweeteners}

In the PCA (principal component analysis) and heat map analysis, sucralose and acesulfame K in Daphnia show ed closer clustering with sodium cylamate, saccharine, and neotame in zebrafish (Fig. 4). Unlike the other compounds, acesulfame K and sucralose in Daphnia increase the heart rate while the other compound decreased the heart rate of daphnia and these effects also observed in zebrafish. Clustering analysis resulted into three groups. The first group comprised of aspartame, alitame, dulcin, neotame, sodium cyclamate, and saccharin showed specific effects on Daphnia. The second group consisting of acesulfame $\mathrm{K}$, alitame, aspartame, dulcin, and sucralose show ed similar effects in zebrafish. Finally, the third group which includes sucralose and acesulfame K in Daphnia and saccharine, neotame, and sodium cyclamate in zebrafish as they have similar effect in altering cardiac parameter endpoint we tested. 

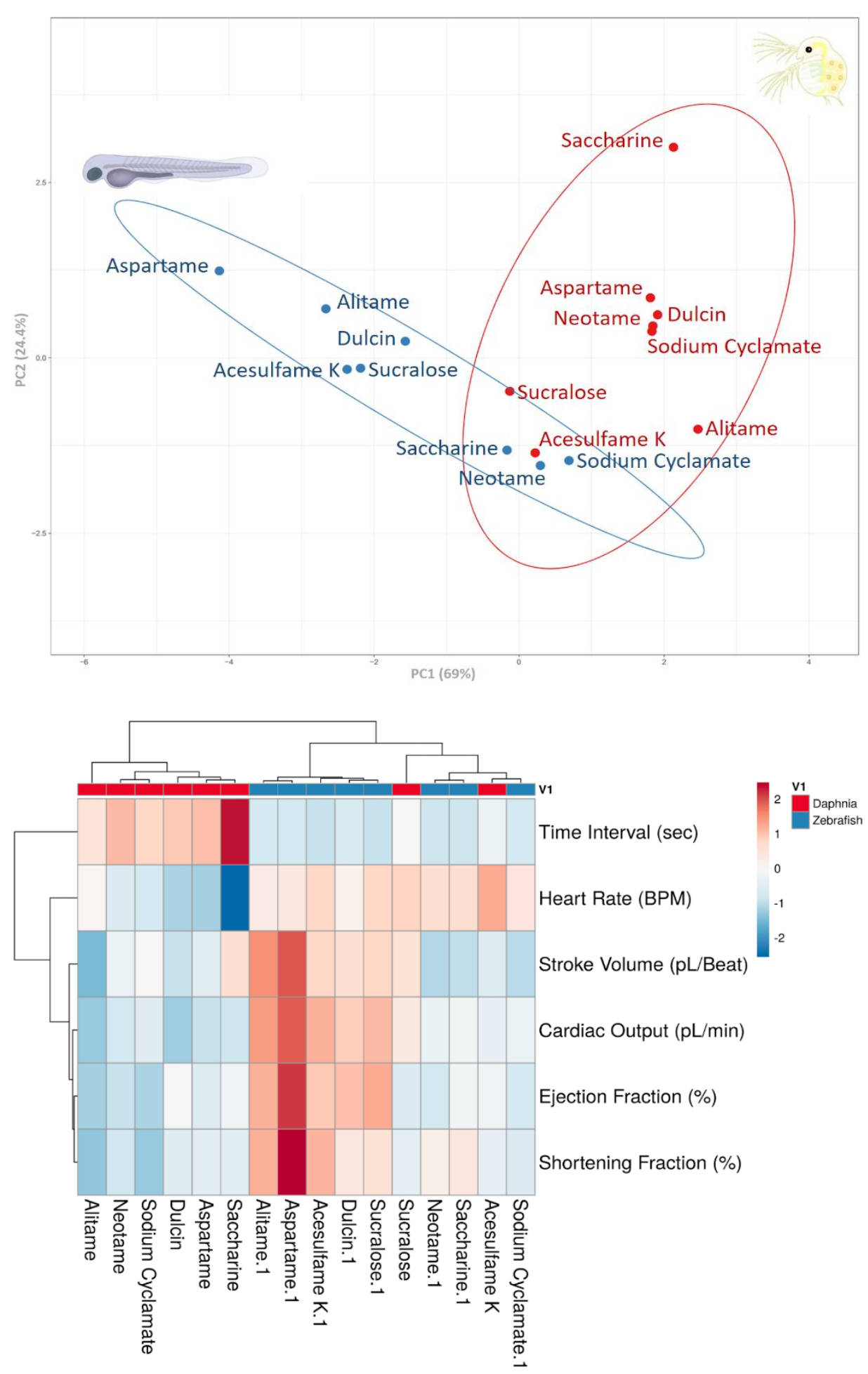

Figure 4. Principal component analysis (Top panel) and Heat map (Bottom panel) for cardiac physiological endpoint alterations in either Daphnia (red color) or zebrafish (blue color) after exposure to eight diffe rent artificial swee teners. Results show the correlation be tween every compound tested in every endpoint. The cardiac physiology endpoints including SV, stroke volume; CO, cardiac output; $\mathrm{EF}$, ejection fractioning; $\mathrm{SF}$, shortening fraction; $\mathrm{HR}$, heart rate and TI, time interval.

\section{Discussion}

4.1. The importance of using zebrafish and Daphnia to study artificial sweetener adverse effects on cardiac performance 
The effects of a number of substances in aquatic animals such as zebrafish, medaka, Daphnia and Xenopus have long been investigated [57-61]. In order to address the full spectrum of toxicity of different substances, studies often utilize multiple aquatic models [62]. Invertebrates such as Daphnia are used for initial toxicity screening followed by more complex organisms such as zebrafish for organ-specific toxicity assessment [62]. Inspired by this idea, we used both Daphnia and zebrafish to test the effects of artificial sw eeteners on cardiac performance parameters.

Based on the PCA and heat map plots, we observed that alitame, aspartame, and dulcin show ed species-specific effects. While receptor-specific binding tests were not performed, we proposed that Daphnia and zebrafish might has different receptors for artificial sweetener ligands. Moreover, in cases where different species have similar receptors for the sameligand, receptors may have different conformations resulting in different responses. This can happen even among species within the similar phylogenetic tree [63-65]. For example, a previous study focused on taste receptors in primates demonstrated that glycine, D-phenylalanine, D-tryptophan, cyanosousan, magapame, and sucronate can induce sweet taste buds to all primates while in contrast, campame, cyclamate, and superaspartameinduced sweet tastebuds only in a few primates [66]. In another study testing pain sensitivity of human show that humans have difference sensitivity to pain because of the variance of pain receptor [67]. These studies suggest that some stimuli have different effects on different species. Therefore, our findings clearly demonstrate zebrafish and Daphnia with distinct response toward artificial sw eeteners on cardiac performance, also support that multiple animal model is required to fully recapitulate the potential adverse effect for artificial sw eeteners in vivo.

\subsection{Saccharin induces distinct cardiac performance in zebrafish and Daphnia}

The most important finding in this study is significant alteration of heart rate and heartbeat interval in zebrafish and Daphnia in an opposite manner by saccharin. A previous study showed that saccharin treatment to rat caused oxidative stress in brain by increasing MDA (Malondialdehyde) level [68]. In previous study, zebrafish treated with saccharin also exhibited infiltration of inflammatory cells into zebrafish liver and brain by increasing cholesteryl ester transfer protein, which play an important role in transporting and exchange of cholesteryl ester to triglyceride and vice versa [20]. Heart rate is controlled by signals from the brain, therefore, damage in the brain may cause alterations in heart rate regulation [69-71].

It $w$ as also reported that saccharin reduced total antioxidant capacity (TAC) and catalase activity and caused liver damage in rats [72]. Furthermore, a separate study also described that saccharin can damage hepatic and renal tissue and significantly decrease catalase, glutathione, and su peroxide dismutase levels in rat liver [73]. Decrease in antioxidant and catalase activity led to the increase of reactive oxygen species levels, which caused tissue damage, especially in sensitive organs such as the heart. In this study, saccharin altered heart rate in both Daphnia and zebrafish. While no evidence of physical damage in the heart w as observed in both species, wehypothesize that oxidative stress may lead to organ damage in longer exposure times. Evidence also showed that incubation in saccharin also caused increase in heart rate variability. While not significant in Daphnia, there was a significant increase in zebrafish heart rate variability which strengthened the possibility of heart damage (Fig. 3).

\subsection{Possible mechanism of artificial sweetener on modulating cardiac performance in zebrafish and Daphnia}

Cardiac function is mediated by the sympathetic and parasympatheticnervous systems [74]. The sympathetic nervous system acts on adrenergic receptors (AR) which are G-Protein coupled receptors (GPCR) [75]. Stimulation of $\beta_{1} A R s$ and $\beta_{2} A R s$ in the heart-increases cardiac contractility, frequency, rate of relaxation, acceleration of impulse conduction through the atrioventricular node as well as increased pacemaker activity from the sinoatrial node by increasing intracellular $\mathrm{Ca}^{2+}$ concentration [76]. Previous studies reported that aspartame can increase brain adrenergic neurotransmitters in various parts of the mouse brain [77]. Furthermore, aspartame increases sympathetic activity within half an hour after consumption either in the form of diluted water or in aspartame-sweetened diet drink in humans [78]. These findings suggest the possibility of artificial sweeteners in altering the 
sympathetic nervous system, and finally induce cardiac performance alteration in zebrafish and Daphnia.

After incubation in acesulfame $\mathrm{K}$, neotame, saccharin, and sucralose, heart rate was significantly elevated in zebrafish. Dopamine is one of hormones that also acts as a neurotransmitter which functions primarily in the central nervous system and usually related to happiness. The role of dopamine on mediating food reward and stimulating palatability is well-established. Sucrose induces dopamine release in rats [79]. Also, the uptake of saccharin increases dopamine levels in rats [80]. Among the reasons for the increase of dopamine release is related with the food rew ard system that is induced by sweet taste [81]. The positive effect of dopamine on heart rate and muscle contractility has been observed in animal models, such as dogs [82] and rats [83], thus corroborating to our results that some artificial sw eeteners can increase heart rate.

$\mathrm{T} 1 \mathrm{R}$ and $\mathrm{T} 2 \mathrm{R}$ are receptors expressing on taste buds that belong to a superfamily of GPCRs mediating sw eet stimuli in humans and arehighly expressed in the olfactory system especially in the tongue $[84,85]$. Those receptors have also been isolated and characterized in fish as well, which share high conservativity to human T1R and T2R counterparts [86]. T1R homolog in zebrafish responds to artificial sweeteners by increasing dopamine concentration [87]. However, although the whole genome of Daphnia magna has been decoded, no report of T1R/T2R taste receptor homologs are found in Daphnia and study by Penalva-Arana et al. suggest that taste response in daphnia meditated by gustatory receptor superfamily (Grs) [88]. Therefore, we proposed this might be one of the reasons to explain why some artificial sw eeteners increase heart rate variability in zebrafish larvaebut not in Daphnia.

$\gamma$-aminobutyric acid (GABA) is one of the neurotransmitters that plays an important role in inhibiting neuronal activity. In vertebrates, the heart rate is controlled by GABA signaling, including in Daphnia and zebrafish [89,90]. For example, the activity of dopamine $\mathrm{D}_{2}$ receptor modulated AKT signaling and altered GABAergic neuron development and motor behavior in zebrafish larvae [91]. Addition of dopamine significantly increased the variability of sd1 in zebrafish larvae [92]. Moreover, GABA receptor homolog in Daphnia pulex showed similar function [93]. Therefore, we hypothesized the heart rate in both zebrafish and Daphnia is mediated by GABA system, and the administration of artificial sw eeteners can trigger similar heart rate variability in zebrafish larvae, yet the lack of sw eet receptors diminished the phenomena in Daphnia.

At the preliminary testing, we found that Daphnia stroke volume and cardiac output have unusually high variation rate. We hypothesize that body size has a positive correlation with heart size and this variation might become a potential bias in accurately assessing 2Dvideo imaging based methods [94]. This problem is not only seen in the assessment of cardiac performance but also affects total protein and other biomarkers in Daphnia [95]. To solve this problem, we normalized the stroke volume and cardiac output by measuring Daphnia body area. Our study also suggests that normalizing data by body size provides a more reliable index especially in cardiac performance analysis [96]. In the future, normalization of stroke volume and cardiac output with the body size should become a good method on accurate assessing cardiac parameters in Daphnia.

\section{Conclusions}

Our study showed the potential adverse effects on cardiac physiology in zebrafish larvae and Daphnia neonates after exposure to commercial artificial sweeteners. Although no significant phenotypic change (like edema) was observed during the experiment, our study demonstrated that acute exposures to artificial sweeteners at low concentration (100 ppb) indeed can alter cardiac performance in two animal models. Our PCA analysis also demonstrated species-specific effects between zebrafish and Daphnia, highlighting the importance of using a variety of animal models in assessing toxicities of chemical compounds.

Author Contributions: Conceptualization, F.S. and C.-D.H.; Data curation, Y.-H.L., H.-T.L. and Z.W .; Funding acquisition and Investigation, J.-C.H. and C.-D.H.; Project administration, C.-D.H.; Resources, H.-T.L.; Supervision, C.-D.H.; Validation and Visualization, Y.-H.L. and F.S.; Writing-original draft, J.-C.H., R.A.T.F., A.P.G.M. and C.-D.H. All authors have read and agreed to the published version of the manuscript. 
Funding: This study was funded by the grants sponsored by the Minis try of Science and Technology MOST 1052313-B-033-001-MY3 and MOST 107-2622-B-033-001-CC2 to C.-D.H.

Acknowledgments: We a pprecia te Freshwater Biore source Center at National Chiayi University on providing water fleas, and Taiwan Zebrafish Stock Center at Aca demia Sinica (TZCAS) on providing zebra fish AB strain.

Conflicts of Interest: The authors declare no conflicts of interest.

\section{References}

1. Burke, M.V.; Small, D.M. Physiological mechanisms by which non-nutritive sweeteners may impact body weight and metabolism. Physiology E behavior 2015, 152, 381-388.

2. Ochoa, M.; Lalles, J.-P.; Malbert, C.-H.; Val-Laillet, D. Dietary sugars: Their de tection by the gut-brain axis and the ir peripheral and central effects in health and dise ases. European journal of nutrition 2015, 54, 1-24.

3. Gardener, H.; Elkind, M.S. Artificial sweeteners, real risks. Am Heart Assoc: 2019.

4. Bian, X.; Chi, L.; Gao, B.; Tu, P.; Ru, H.; Lu, K. The artificial sweetener a cesulfame potassium affects the gut microbiome and body weight gain in cd-1 mice. PLoS One 2017, 12, e0178426.

5. Swithers, S.E. Artificial sweeteners produce the counterintuitive effect of inducing metabolic derangements. Trends in Endocrinology \& Metabolism 2013, 24, 431-441.

6. Katakami, N. Mechanism of development of atherosclerosis and cardiovascular disease in diabetes mellitus. Journal of atherosclerosis and thrombosis 2017, RV17014.

7. Beckman, J.A.; Creager, M.A.; Libby, P. Diabetes and a therosclerosis: Epidemiology, pathophysiology, and management. Jama 2002, 287, 2570-2581.

8. Al-Eisa, R.A.; Al-Salmi, F.A.; Hamza, R.Z.; El-Shenawy, N.S. Role of l-carnitine in protection against the cardiac oxida tive stress induce d by a spartame in wistar albino rats. PloS one 2018, 13, e0204913.

9. Choudhary, A.K.; Sundareswaran, L.; Devi, R.S. Effects of aspartame on the evaluation of electrophysiological re sponses in wistar albino rats. Journal of Taibah University for Science 2016, 10, 505-512.

10. Saucedo-Vence, K.; Elizalde-Velázquez, A.; Dublán-García, O.; Galar-Martínez, M.; Islas-Flores, H.; SanJuan-Reyes, N.; García-Medina, S.; Hernández-Navarro, M.D.; Gómez-Oliván, L.M. Toxicological hazard induce d by sucralose to environmentally relevant concentrations in common carp (cyprinus carpio). Science of the Total Environment 2017, 575, 347-357.

11. Azad, M.B.; Abou-Setta, A.M.; Chauhan, B.F.; Rabbani, R.; Lys, J.; Copstein, L.; Mann, A.; Jeyaraman, M.M.; Reid, A.E.; Fiander, M. Nonnutritive sweeteners and cardiometabolic health: A systematic review and meta-analysis of randomized controlled trials and prospective cohort studies. Cmaj 2017, 189, E929-E939.

12. Negro, F.; Mondardini, A.; Palmas, F. Hepatotoxicity of saccharin. New England Journal of Medicine 1994, 331, 134-135.

13. Blumenthal, H.J.; Vance, D.A. Chewing gum headaches. Headache: The Journal of Head and Face Pain 1997, 37, 665-666.

14. Cadirci, K.; Tozlu, O.O.; Türkez, H.; Mardinoglu, A. The in vitro cytotoxic, genotoxic, and oxidative damage potentials of the oral artificial sweetener a spartame on culture d human blood cells. Turkish Journal of Medical Sciences 2020, 50, 448-454.

15. Tsakiris, S.; Giannoulia-Ka rantana, A.; Simintzi, I.; Schulpis, K.H. The effect of aspartame metabolites on human erythrocyte membrane a cetylcholine sterase activity. Pharmacolog ical research 2006, 53, 1-5.

16. Risdon, S.; Meyer, G.; Marziou, A.; Riva, C.; Roustit, M.; Walther, G. Artificial sweeteners impair endothelial vascular reactivity: Preliminary results in rodents. Nutrition, Metabolism and Cardiovascular Diseases 2020, 843-846.

17. Suez, J.; Korem, T.; Zeevi, D.; Zilberman-Schapira, G.; Thaiss, C.A.; Maza, O.; Israeli, D.; Zmora, N.; Gilad, S.; Weinberger, A. Artificial sweeteners induce glucose intole rance by altering the gut microbiota. Natume 2014, 514, 181-186.

18. Whitehouse, C.R.; Boullata, J.; McCauley, L.A. The potential toxicity of artificial sweeteners. Aaohn Journal 2008, 56, 251-261.

19. Yin, K.-J.; Xie, D.-Y.; Zhao, L.; Fan, G.; Ren, J.-N.; Zhang, L.-L.; Pan, S.-Y. Effects of diffe rent sweeteners on behavior and neurotransmitters release in mice. Journal of Food Science and Technology 2020, 57, 113-121.

20. Kim, J.-Y.; Seo, J.; Cho, K.-H. Aspartame-fed zebrafish exhibit acute deaths with swimming defects and saccharin-fed zebrafish have elevation of chole steryl ester transfer protein activity in hype rcholesterolemia. Food and Chemical Toxicology 2011, 49, 2899-2905. 
21. Weerasooriyagedara, M. Toxicity effects of aspartame on embryonic de velopment of zebrafish (danio re rio). International Journal of Engineering and Management Research (IJEMR) 2018, 8, 183-188.

22. Wiklund, A.-K.E.; Adolfsson-Erici, M.; Liewenborg, B.; Gorokhova, E. Sucralose induces biochemical responses in daphnia magna. PloS one 2014, 9, e92771.

23. Giardoglou, P.; Beis, D. On zebrafish disease models and matters of the heart. Biomedicines 2019, 7, 15.

24. Gut, P.; Reischauer, S.; Stainier, D.Y.; Arnaout, R. Little fish, big data: Zebrafish as a model for cardiova scular and metabolic dise ase. Physiological reviews 2017, 97, 889-938.

25. Asnani, A.; Peterson, R.T. The zebrafish a s a tool to identify novel the rapies for human cardiovascular disease. Diseasemodels E mechanisms 2014, 7, 763-767.

26. Poon, K.L.; Brand, T. The zebrafish model system in cardiovascular research: A tiny fish with mighty prospects. Global Cardiology Science and Practice 2013, 2013, 4.

27. Benslimane, F.M.; Alser, M.; Zakaria, Z.Z.; Sharma, A.; Abdelrahman, H.A.; Yalcin, H.C. Adaptation of a mice doppler echocardiography platform to measure cardiac flow velocities for embryonic chicken and a dult zebrafish. Frontiers in bioeng ineering and biotechnology 2019, 7, 96.

28. Lal, H.; Misra, V.; Viswana than, P.; Murti, C.K. The water flea (da phnia magna) as a sensitive indica tor for the assessment of toxicity of synthe tic de tergents. Ecotoxicology and environmental safety 1984, 8, 447-450.

29. Zhu, X.; Chang, Y.; Chen, Y. Toxicity and bioaccumulation of tio2 nanoparticle aggregates in daphnia magna. Chemosphere 2010, 78, 209-215.

30. Whiteoak, A.M.; Penson, P.E. 220 da phnia magna as a model for quantifying cha os in cardiac arrhythmia. Heart 2017, 103, A143-A143.

31. Sarasamma, S.; Audira, G.; Siregar, P.; Malhotra, N.; Lai, Y.-H.; Liang, S.-T.; Chen, J.-R.; Chen, K.H.-C.; Hsiao, C.-D. Nanoplastics cause neurobehavioral impairments, reproductive and oxida tive damages, and biomarker responses in zebrafish: Throwing up alarms of wide spread health risk of exposure. International Journal of Molecular Sciences 2020, 21, 1410.

32. Hussain, A.; Audira, G.; Siregar, P.; Lin, Y.-C.; Villalobos, O.; Villaflores, O.; Wang, W.-D.; Hsiao, C.-D. Waterborne exposure of paclobutrazol at environmental relevant concentration induce locomotion hyperactivity in larvae and anxiolytic exploratory behavior in adult zebrafish. International Journal of Environmental Research and Public Health 2020, 17, 4632.

33. Bui Thi, N.H.; Nguyen Thi, N.A.; Audira, G.; Siregar, P.; Liang, S.-T.; Huang, J.-C.; Hsiao, C.-D. Chronic exposure to low concentration lead chloride-induced anxiety and loss of aggression and memory in ze brafish. International journal of molecular sciences 2020, 21, 1844.

34. Avdesh, A.; Chen, M.; Martin-Iverson, M.T.; Mondal, A.; Ong, D.; Rainey-Smith, S.; Taddei, K.; Lardelli, M.; Groth, D.M.; Verdile, G. Regular care and maintenance of a zebrafish (danio rerio) laboratory: An introduction. JoVE (Journal of Visualized Experiments) 2012, e4196.

35. Bunescu, A.; Garric, J.; Vollat, B.; Canet-Soulas, E.; Graveron-Demilly, D.; Fauvelle, F. In vivo proton hrmas nmr metabolic profile of the freshwater cladoceran da phnia magna. Molecular BioSystems 2010, 6, 121125.

36. Sánchez-Vázquez, F.J.; Terry, M.I.; Felizardo, V.O.; Vera, L.M. Daily rhythms of toxicity and effectiveness of ane sthetics (ms222 and eugenol) in zebra fish (danio rerio). Chronobiology international 2011, 28, 109-117.

37. Monnard, C.R.; Grasser, E.K. Perspective: Cardiovascular responses to sugar-sweetened beverages in humans: A narrative review with potential hemodynamic mechanisms. Advances in Nutrition 2018, 9, 7077.

38. Grasser, E.K.; Dulloo, A.; Montani, J.-P. Cardiovascular re sponses to the inge stion of sugary drinks using a randomised cross-over study design: Does glucose attenuate the blood pressure-elevating effect of fructose? British journal of nutrition 2014, 112, 183-192.

39. Santoso, F.; Sampurna, B.P.; Lai, Y.-H.; Liang, S.-T.; Hao, E.; Chen, J.-R.; Hsiao, C.-D. Development of a simple imagej-based method for dynamic blood flow tracking in zebra fish embryos and its application in drug toxicity evaluation. Inventions 2019, 4, 65.

40. Sampurna, B.P.; Audira, G.; Juniardi, S.; Lai, Y.-H.; Hsiao, C.-D. A simple imagej-based method to measure cardiac rhythm in zebra fish embryos. Inventions 2018, 3, 21.

41. Rueden, C.T.; Schindelin, J.; Hiner, M.C.; DeZonia, B.E.; Walter, A.E.; Arena, E.T.; Eliceiri, K.W. Imagej2: Imagej for the next generation of scientific image data. BMC bioinformatics 2017, 18, 529.

42. Hoage, T.; Ding, Y.; Xu, X. Quantifying cardiac functions in embryonic and adult zebrafish. In Cardiovascular development, Springer: 2012; pp 11-20. 
43. Metsalu, T.; Vilo, J. Clustvis: A web tool for visualizing clustering of multivariate data using principal component analysis and he atmap. Nucleic acids research 2015, 43, W566-W570.

44. Mirescu, Ş.; Harden, S. Nonlinear dynamics methods for assessing heart rate variability in patients with recentmyocardial infarction. Rom. J. Biophys 2012, 22, 117-124.

45. Waselius, T.; Wikgren, J.; Halkola, H.; Penttonen, M.; Nokia, M.S. Learning by heart: Cardiac cycle reveals an effective time window for le arning. Journal of Neurophysiology 2018, 120, 830-838.

46. Feng, H.-Z.; Jin, J.-P. A protocol to study ex vivo mouse working heart at human-like heart rate. Journal of molecular and cellular cardiology 2018, 114, 175-184.

47. Kinsara, A.J.; Najm, H.K.; Al Anazi, M.; Ta mim, H. Resting he art rate in pa tients with is che mic heart disease in saudi arabia and egypt. Journal of the Saudi Heart Association 2011, 23, 225-232.

48. Gomez, O.; Okumura, K.; Honjo, O.; Sun, M.; Ishii, R.; Bijnens, B.; Friedberg, M.K. Heart rate reduction improves biventricular function and interactions in experimental pulmonary hypertension. American Journal of Physiology-Heart and Circulatory Physiology 2018, 314, H542-H551.

49. McGrath, P. Zebrafish: Methods for assessing drug safety and toxicity. John Wiley \& Sons: 2012.

50. Ehrman, J.K.; Kerrigan, D.; Keteyian, S. Advanced exercise physiology: Essential concepts and applications. Human Kinetics: 2018.

51. Dunn, J.-O.; Mythen, M.; Grocott, M. Physiology of oxygen transport. Bja Education 2016, 16, 341-348.

52. Costanzo, L.S. Physiology e-book. Elsevier Health Sciences: 2017.

53. Mourot, L.; Bouhaddi, M.; Perrey, S.; Rouillon, J.-D.; Regnard, J. Quantitative poincare plot analysis of heart rate variability: Effect of endurance training. European journal of applied physiology 2004, 91, 79-87.

54. Chan, P.K.; Lin, C.C.; Cheng, S.H. Noninvasive technique for measurement of heartbeat regularity in zebrafish (danio re rio) e mbryos. BMC biotechnology 2009, 9, 11.

55. Charlet, A.; Rodeau, J.-L.; Poisbeau, P. Poincare plot descriptors of heart rate variability as markers of persistent pain expression in freely moving rats. Physiology E behavior 2011, 104, 694-701.

56. Liu, J.; Wei, W.; Kuang, H.; Tsien, J.Z.; Zhao, F. He art ra te and he art ra te va riability assessment identifies individual differences in fear response magnitudes to earthquake, free fall, and air puff in mice. PLoS One 2014, 9, e93270.

57. Kim, J.-Y.; Park, K.-H.; Kim, J.; Choi, I.; Cho, K.-H. Modified high-density lipoproteins by artificial swee tener, a spartame, and sa ccharin, showed loss of anti-a therosclerotic activity and toxicity in zebra fish. Cardiovascular toxicology 2015, 15, 79-89.

58. Graffin, R. Effects of sucralose, saccharin, rebaudioside (in stevia) and aspartame on development in xenopus laevis (clawed frog). 2016.

59. Selderslaghs, I.W.; Blust, R.; Witters, H.E. Fea sibility study of the zebra fish as say as an alte rnative me thod to screen for developmental toxicity and embryotoxicity using a training set of 27 compounds. Reproductive toxicology 2012, 33, 142-154.

60. Lee, W.; Wang, Y.-C. Assessing developmental toxicity of caffeine and sweeteners in medaka (oryzias latipes). SpringerPlus 2015, 4, 1-10.

61. Huggett, D.; Stoddard, K. Effects of the artificial sweetener sucralose on da phnia magna and a mericamysis bahia survival, growth and re production. Food and chemical toxicology 2011, 49, 2575-2579.

62. Jang, G.H.; Park, C.-B.; Kang, B.J.; Kim, Y.J.; Lee, K.H. Sequential a ssessment via da phnia and zebrafish for systematic toxicity screening of he terogeneous substances. Environmental Pollution 2016, 216, 292-303.

63. Jeyanthi, G.P. Molecular biology. MJP Publisher: Chennai, India, 2019.

64. Carson, S.; Miller, H.B.; Srougi, M.C.; Witherow, D.S. Molecular biology techniques: A classroom laboratory manual. Else vier Science: 2019.

65. Lohar, P.S. Cell and molecular biology. MJP Publisher: 2019.

66. Nofre, C.; Tinti, J.; Glaser, D. Evolution of the sweetness receptor in primates. Ii. Gustatory responses of non-human primates to nine compounds known to be sweet in man. Chemical senses 1996, 21, 747-762.

67. Young, E.E.; Lariviere, W.R.; Belfer, I. Genetic basis of pain variability: Recent advances. Journal of medical genetics 2012, 49, 1-9.

68. Erbaş, O.; Erdoğan, M.A.; Khalilnezhad, A.; Solmaz, V.; Gürkan, F.T.; Yiğittürk, G.; Eroglu, H.A.; Taskiran, D. Evaluation of long-term effects of artificial sweeteners on rat brain: A biochemical, behavioral, and histological study. Journal of biochemical and molecular toxicology 2018, 32, e22053. 
69. Lacquaniti, L.G.; Irone, M.; Barbacini, S.; Merlo, F.; Demo, P.; Pellegrin, C.; Dan, M. Heart rate va riability and severe brain damage: Preliminary data. International journal of clinical monitoring and computing 1993, 10, 181-185.

70. Ikeda, T.; Murata, Y.; Quilligan, E.J.; Parer, J.T.; Theunissen, I.M.; Cifuentes, P.; Doi, S.; Park, S.-D. Fetal heart rate patterns in postasphyxiated fetal lambs with brain damage. American journal of obstetrics and gynecology 1998, 179, 1329-1337.

71. Savenije, B.; Lambooij, E.; Gerritzen, M.; Korf, J. Development of brain damage as measured by brain impedance recordings, and changes in heart rate, and blood pressure induced by different stunning and killing methods. Poultry science 2002, 81, 572-578.

72. Alkafafy, M.E.-S.; Ibrahim, Z.S.; Ahmed, M.M.; El-Shazly, S.A. Impact of aspartame and saccharin on the rat liver: Biochemical, molecular, and histological a pproach. International journal of immunopathology and pharmacology 2015, 28, 247-255.

73. Amin, K.A.; Al-Muzafar, H.M.; Elsttar, A.H.A. Effect of sweetener and fla voring agent on oxida tive indices, liver and kidney function levels in rats. 2016.

74. Gordan, R.; Gwa thmey, J.K.; Xie, L.-H. Autonomic and endocrine control of cardiovascular function. Wond journal of cardiology 2015, 7, 204.

75. Brodal, P. The central nervous system. Oxford University Press: 2010.

76. Lymperopoulos, A.; Rengo, G.; Koch, W.J. Adrenergic nervous system in heart failure: Pathophysiology and the rapy. Circulation research 2013, 113, 739-753.

77. Coulombe Jr, R.A.; Sharma, R.P. Neurobiochemical alterations induced by the artificial sweetener as partame (nutra sweet). Toxicology and applied pharmacology 1986, 83, 79-85.

78. Priya, K.A.; Prasath, G.S. Comparison of effect of aspartame (artificial sweete ner) and aspartamesweetened diet drink on autonomic reactivity of volunteers. National Journal of Physiology, Pharmacy and Pharmacology 2018, 8, 1057-1060.

79. De Araujo, I.E.; Oliveira-Maia, A.J.; Sotnikova, T.D.; Gainetdinov, R.R.; Caron, M.G.; Nicolelis, M.A.; Simon, S.A. Food reward in the absence of taste receptor signaling. Neuron 2008, 57, 930-941.

80. Mark, G.; Blander, D.; Hoebel, B. A conditioned stimulus decreases extracellular dopamine in the nucleus accumbens after the de velopment of a le arned taste a version. Brain research 1991, 551, 308-310.

81. De Araujo, I.E. Sweet taste signaling and the formation of memories of energy sources. Frontiers in systems neuroscience 2011, 5, 99.

82. Holmes, J.C.; Fowler, N.O. Direct cardia ceffects of dopa mine. Circulation research 1962, 10, 68-72.

83. Ferrari, P.; Van Erp, A.; Tornatzky, W.; Miczek, K. Accumbal dopamine and serotonin in anticipation of the next aggressive episode in rats. European Journal of Neuroscience 2003, 17, 371-378.

84. Bachmanov, A.A.; Beauchamp, G.K. Taste receptor genes. Annu. Rev. Nutr. 2007, 27, 389-414.

85. Nelson, G.; Hoon, M.A.; Chandrashekar, J.; Zhang, Y.; Ryba, N.J.; Zuker, C.S. Mammalian sweet taste rece ptors. Cell 2001, 106, 381-390.

86. Ishima ru, Y.; Okada, S.; Naito, H.; Nagai, T.; Yasuoka, A.; Matsumoto, I.; Abe, K. Two familie s of candidate taste receptors in fishes. Mechanisms of development 2005, 122, 1310-1321.

87. Oike,H.; Nagai, T.; Furuyama, A.; Okada, S.; Aihara, Y.; Ishimaru, Y.; Marui, T.; Matsumoto, I.; Misaka, T.; Abe, K. Characterization of ligands for fish taste receptors. Journal of Neuroscience 2007, 27, 5584-5592.

88. Peñalva-Arana, D.C.; Lynch, M.; Robertson, H.M. The chemoreceptorgenes of the waterflea da phnia pulex: Many grs but no ors. BMC evolutionary biology 2009, 9, 79.

89. Antonaccio, M.J.; Taylor, D.G. Involvement of central gaba receptors in the regulation of blood pressure and heart rate of anesthetized cats. European journal of pharmacology 1977, 46, 283-287.

90. Williford, D.J.; Hamilton, B.L.; Souza, J.D.; Williams, T.P.; Dimicco, J.A.; Gillis, R.A. Centralne rvous system mechanisms involving gaba influence arterial pressure and he art rate in the cat. Circulation research 1980, $47,80-88$.

91. Souza, B.R.; Romano-Silva, M.A.; Tropepe, V. Dopamine d2 receptor activity modula tes akt signa ling and alters gabaergic neuron de velopment and motor behavior in zebrafish larvae. Journal of Neuroscience 2011, 31, 5512-5525.

92. Vargas, R.A. Effects of gaba, neural regulation, and intrinsic cardiac factors on heart rate variability in zebrafish larvae. Zebrafish 2017, 14, 106-117. 
93. McCoole, M.D.; D' Andrea, B.T.; Baer, K.N.; Christie, A.E. Genomic analyses of gas (nitric oxide and carbon monoxide) and small molecule transmitter (acetylcholine, glutamate and gaba) signaling systems in daphnia pulex. Comparative Biochemistry and Physiology Part D: Genomics and Proteomics 2012, 7, 124-160.

94. Bäumer, C.; Pirow, R.; Paul, R. Circulatory oxygen transport in the water flea daphnia magna. Journal of Comparative Physiology B 2002, 172, 275-285.

95. Wiklund, A.-K.E.; Adolfsson-Erici, M.; Liewenborg, B.; Gorokhova, E. Sucralose induces biochemical responses in da phnia magna. PloS one 2014, 9.

96. Dewey, F.E.; Rosenthal, D.; Murphy Jr, D.J.; Froelicher, V.F.; Ashley, E.A. Does size matter? Clinical applications of scaling cardiac size and function for body size. Circulation 2008, 117, 2279-2287. 